\title{
Time, Space, and Energy in Reversible Computing
}

\author{
Paul Vitányi ${ }^{*}$ \\ University of Amsterdam \\ National ICT of Australia
}

\begin{abstract}
We survey results of a quarter century of work on computation by reversible general-purpose computers (in this setting Turing machines), and general reversible simulation of irreversible computations, with respect to energy-, time- and space requirements.

Categories and Subject Descriptors: F.2 [Algorithms], F.1.3 [Performance]

General Terms: Algorithms, Performance

Keywords: Reversible computing, reversible simulation, adiabatic computing, low-energy computing, computational complexity, time complexity, space complexity, energy dissipation complexity, tradeoffs.
\end{abstract}

\section{INTRODUCTION}

Computer power has roughly doubled every 18 months for the last half-century (Moore's law). This increase in power is due primarily to the continuing miniaturization of the elements of which computers are made, resulting in more and more elementary gates per unit area with higher and higher clock frequency, accompanied by less and less energy dissipation per elementary computing event. Roughly, a linear increase in clock speed is accompanied by a square increase in elements per unit area-so if all elements compute all of the time, then the dissipated energy per time unit rises cubicly (linear times square) in absence of energy decrease per elementary event. The continuing dramatic decrease in dissipated energy per elementary event is what has made Moore's law possible. But there is a foreseeable end to this: There is a minimum quantum of energy dissipation associated with elementary events. This puts a fundamental limit on how far we can go with miniaturization, or does it?

R. Landauer [11] has demonstrated that it is only the 'logically irreversible' operations in a physical computer that necessarily dis-

\footnotetext{
*Part of this work was done while the author was on sabbatical leave at National ICT of Australia, Sydney Laboratory at UNSW. Supported in part by the EU Project RESQ IST-2001-37559, the ESF QiT Programmme, the EU NoE PASCAL, and the Netherlands BSIK/BRICKS project. Address: CWI, Kruislaan 413, 1098 SJ Amsterdam, The Netherlands. Email: Paul.Vitanyi@cwi.nl
}

Permission to make digital or hard copies of all or part of this work for personal or classroom use is granted without fee provided that copies are not made or distributed for profit or commercial advantage and that copies bear this notice and the full citation on the first page. To copy otherwise, to republish, to post on servers or to redistribute to lists, requires prior specific permission and/or a fee.

CF'05, May 4-6, 2005, Ischia, Italy.

Copyright 2005 ACM 1-59593-018-3/05/0005 ...\$5.00. sipate energy by generating a corresponding amount of entropy for every bit of information that gets irreversibly erased; the logically reversible operations can in principle be performed dissipation-free. One should sharply distinguish between the issue of logical reversibility and the issue of energy dissipation freeness. If a computer operates in a logically reversible manner, then it still may dissipate heat. For such a computer we know that the laws of physics do not preclude that one can invent a technology in which to implement a logically similar computer to operate physically in a dissipationless manner. Computers built from reversible circuits, or the reversible Turing machine, [1] 2] 7], implemented with current technology will presumably dissipate energy but may conceivably be implemented by future technology in an adiabatic fashion. But for logically irreversible computers adiabatic implementation is widely considered impossible.

Thought experiments can exhibit a computer that is both logically and physically perfectly reversible and hence perfectly dissipationless. An example is the billiard ball computer, [7], and similarly the possibility of a coherent quantum computer, [6, 19]. Our purpose is to determine the theoretical ultimate limits to which the irreversible actions in an otherwise reversible computation can be reduced.

Currently, computations are commonly irreversible, even though the physical devices that execute them are fundamentally reversible. At the basic level, however, matter is governed by classical mechanics and quantum mechanics, which are reversible. This contrast is only possible at the cost of efficiency loss by generating thermal entropy into the environment. With computational device technology rapidly approaching the elementary particle level it has been argued many times that this effect gains in significance to the extent that efficient operation (or operation at all) of future comput-

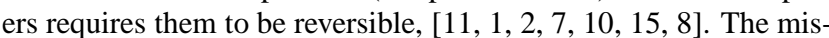
match of computing organization and reality will express itself in friction: computers will dissipate a lot of heat unless their mode of operation becomes reversible, possibly quantum mechanical. Since 1940 the dissipated energy per bit operation in a computing device has - with remarkable regularity-decreased at the inverse rate of Moore's law [10] (making Moore's law possible). Extrapolation of current trends shows that the energy dissipation per binary logic operation needs to be reduced below $k T$ (thermal noise) within 20 years. Here $k$ is Boltzmann's constant and $T$ the absolute temperature in degrees Kelvin, so that $k T \approx 3 \times 10^{-21}$ Joule at room temperature. Even at $k T$ level, a future device containing 1 trillion $\left(10^{12}\right)$ gates operating at 1 terahertz $\left(10^{12}\right)$ switching all gates all of the time dissipates about 3000 watts. Consequently, in contemporary computer and chip architecture design the issue of power consumption has moved from a background worry to a major problem. Theoretical advances in reversible computing are scarce and 
far between; many serious ones are listed in the references. For this review we have drawn primarily on the results and summarized material in [15 17 5]. It is a tell-tale sign of the difficulty of this area, that no further advances have been made in this important topic since that time.

\section{REVERSIBLE TURING MACHINES}

There is a decisive difference between reversible circuits and reversible special purpose computers [7| on the one hand, and reversible universal computers on the other hand [1, 3]. While one can design a special-purpose reversible version for every particular irreversible circuit using reversible universal gates, such a method does not yield an irreversible-to-reversible compiler that can execute any irreversible program on a fixed universal reversible computer architecture as we are interested in here.

In the standard model of a Turing machine the elementary operations are rules in quadruple format $(p, s, a, q)$ meaning that if the finite control is in state $p$ and the machine scans tape symbol $s$, then the machine performs action $a$ and subsequently the finite control enters state $q$. Such an action $a$ consists of either printing a symbol $s^{\prime}$ in the tape square scanned, or moving the scanning head one tape square left or right.

Quadruples are said to overlap in domain if they cause the machine in the same state and scanning the same symbol to perform different actions. A deterministic Turing machine is defined as a Turing machine with quadruples no two of which overlap in domain.

Now consider the special format (deterministic) Turing machines using quadruples of two types: read/write quadruples and move quadruples. A read/write quadruple $(p, a, b, q)$ causes the machine in state $p$ scanning tape symbol $a$ to write symbol $b$ and enter state $q$. A move quadruple $(p, *, \sigma, q)$ causes the machine in state $p$ to move its tape head by $\sigma \in\{-1,+1\}$ squares and enter state $q$, oblivious to the particular symbol in the currently scanned tape square. (Here ' -1 ' means 'one square left', and ' +1 ' means 'one square right'.) Quadruples are said to overlap in range if they cause the machine to enter the same state and either both write the same symbol or (at least) one of them moves the head. Said differently, quadruples that enter the same state overlap in range unless they write different symbols. A reversible Turing machine is a deterministic Turing machine with quadruples no two of which overlap in range. A $k$-tape reversible Turing machine uses $(2 k+2)$ tuples which, for every tape separately, select a read/write or move on that tape. Moreover, any two tuples can be restricted to some single tape where they don't overlap in range.

Simulation of irreversible Turing machines by reversible ones goes back to Lecerf [13] in 1963, a paper that was little noticed and only recently rediscovered, and independently Bennett [1] in 1973, which paper commonly gets all the credit. To show that every partial recursive function can be computed by a reversible Turing machine one can proceed as follows [1]. Take the standard irreversible Turing machine computing that function. We modify it by adding an auxiliary storage tape called the 'history tape'. The quadruple rules are extended to 6-tuples to additionally manipulate the history tape. To be able to reversibly undo (retrace) the computation deterministically, the new 6-tuple rules have the effect that the machine keeps a record on the auxiliary history tape consisting of the sequence of quadruples executed on the original tape. Reversibly undoing a computation entails also erasing the record of its execution from the history tape. This notion of reversible computation means that only $1: 1$ recursive functions can be computed. To reversibly simulate an irreversible computation from $x$ to $f(x)$ one reversibly computes from input $x$ to output $\langle x, f(x)\rangle$.
Reversible Turing machines or other reversible computers will require special reversible programs. One feature of such programs is that they should be executable when read from bottom to top as well as when read from top to bottom. Examples are the programs in the later sections. In general, writing reversible programs will be difficult. However, given a general reversible simulation of irreversible computation, one can simply write an oldfashioned irreversible program in an irreversible programming language, and subsequently simulate it reversibly. This leads to the following:

DEFINITION 1. An irreversible-to-reversible compiler receives an irreversible program as input and compiles it to a reversible program.

\section{ADIABATIC COMPUTATION}

All computations can be performed logically reversibly, [1], at the cost of eventually filling up the memory with unwanted garbage information. This means that reversible computers with bounded memories require in the long run irreversible bit operations, for example, to erase records irreversibly to create free memory space. The minimal possible number of irreversibly erased bits to do so is believed to determine the ultimate limit of heat dissipation of the computation by Landauer's principle, [11 11 2]. In reference [4] we and others developed a mathematical theory for the unavoidable number of irreversible bit operations in an otherwise reversible computation.

Methods to implement (almost) reversible and dissipationless computation using conventional technologies are often designated by the catch phrase 'adiabatic switching'. Many currently proposed physical schemes implementing adiabatic computation reduce irreversibility by using longer switching times. This is done typically by switching over equal voltage gates after voltage has been equalized slowly. This type of switching does not dissipate energy, the only energy dissipation is incurred by pulling voltage up and down: the slower it goes the less energy is dissipated. If the computation goes infinitely slow, zero energy is dissipated. Clearly, this counteracts the purpose of low energy dissipation which is faster computation.

In [15] it is demonstrated that even if adiabatic computation technology advances to switching with no time loss, a similar phenomenon arises when we try to approach the ultimate limits of minimal irreversibility of an otherwise reversible computation, and hence minimal energy dissipation. This time the effect is due to the logical method of reducing the number of irreversible bit erasures in the computation irrespective of individual switching times. By computing longer and longer (in the sense of using more computation steps), the amount of dissipated energy gets closer to ultimate limits. Moreover, one can trade-off time (number of steps) for energy: there is a new time-irreversibility (time-energy) tradeoff hierarchy. The bounds we derive are also relevant for quantum computations which are reversible except for the irreversible observation steps, 20 19.

\subsection{Background}

Around 1940 a computing device dissipated about $10^{-2}$ Joule per bit operation at room temperature. Since that time the dissipated energy per bit operation has roughly decreased by one order of magnitude (tenfold) every five years. Currently, a bit operation dissipates ${ }^{1}$ about $10^{-17}$ Joule.

Considerations of thermodynamics of computing started in the early fifties. J. von Neumann reputedly thought that a computer

\footnotetext{
${ }^{1}$ After R.W. Keyes, IBM Research.
} 
operating at temperature $T$ must dissipate at least $k T \ln 2$ Joule per elementary bit operation (about $3 \times 10^{-21} \mathrm{~J}$ at room temperature).

Around 1960, R. Landauer [11] more thoroughly analyzed this question and concluded that it is only 'logically irreversible' operations that dissipate energy. An operation is logically reversible if its inputs can always be deduced from the outputs. Erasure of information in a way such that it cannot be retrieved is not reversible. Erasing each bit costs $k T \ln 2$ energy, when computer operates at temperature $T$.

\subsection{Irreversibility Cost of Computation}

The ultimate limits of energy dissipation by computation will be expressed in number of irreversibly erased bits. Hence we consider compactification of records. In analogy of garbage collection by a garbage truck, the cost is less if we compact the garbage before we throw it away. The ultimate compactification which can be effectively exploited is expressed in terms of Kolmogorov complexity.

Technically, the Kolmogorov complexity of $x$ given $y$ is the length of the shortest binary program, for the reference universal prefix Turing machine, that on input $y$ outputs $x$; it is denoted as $C(x \mid y)$. For precise definitions, theory and applications, see [16]. The Kolmogorov complexity of $x$ is the length of the shortest binary program with no input that outputs $x$; it is denoted as $C(x)=C(x \mid \lambda)$ where $\lambda$ denotes the empty input. Essentially, the Kolmogorov complexity of a file is the length of the ultimate compressed version of the file.

Let $\mathbf{R}=R_{1}, R_{2}, \ldots$ be a standard enumeration of reversible Turing machines, [1]. We define $E(\cdot, \cdot)$ as in [4] (where it is denoted as $\left.E_{3}(\cdot, \cdot)\right)$.

DEFINITION 2. The irreversibility cost $E_{R}(x, y)$ of computing $y$ from $x$ by a reversible Turing machine $R$ is is

$$
E_{R}(x, y)=\min \{|p|+|q|: R(\langle x, p\rangle)=\langle y, q\rangle\} .
$$

We denote the class of all such cost functions by $\mathcal{E}$.

We call an element $E_{Q}$ of $\mathcal{E}$ a universal irreversibility cost function, if $Q \in \mathbf{R}$, and for all $R$ in $\mathbf{R}$

$$
E_{Q}(x, y) \leq E_{R}(x, y)+c_{R},
$$

for all $x$ and $y$, where $c_{R}$ is a constant which depends on $R$ but not on $x$ or $y$. Standard arguments from the theory of Turing machines show the following.

LEMMA 1. There is a universal irreversibility cost function in E. Denote it by $E_{U R}$.

PROOF. In [1] a universal reversible Turing machine $U R$ is constructed which satisfies the optimality requirement.

Two such universal (or optimal) machines $U R$ and $U R^{\prime}$ will assign the same irreversibility cost to a computation apart from an additive constant term $c$ which is independent of $x$ and $y$ (but does depend on $U R$ and $U R^{\prime}$ ). We select a reference universal function $U R$ and define the irreversibility cost $E(x, y)$ of computing $y$ from $x$ as

$$
E(x, y) \equiv E_{U R}(x, y) .
$$

Because of the expression for $E(x, y)$ in Theorem 1 below it is called the sum cost measure in [4].

In physical terms this cost is in units of $k T \ln 2$, where $k$ is Boltzmann's constant, $T$ is the absolute temperature in degrees Kelvin, and $\ln$ is the natural logarithm.

Because the computation is reversible, this definition is symmetric: we have $E(x, y)=E(y, x)$.
In our definitions we have pushed all bits to be irreversibly provided to the start of the computation and all bits to be erased to the end of the computation. It is easy to see that this is no restriction. If we have a computation where irreversible acts happen throughout the computation, then we can always mark the bits to be erased, waiting with actual erasure until the end of the computation. Similarly, the bits to be provided can be provided (marked) at the start of the computation while the actual reading of them (simultaneously unmarking them) takes place throughout the computation).

Now let us consider a general computation which outputs string $y$ from input string $x$. We want to know the minimum irreversibility cost for such computation. This leads to the following theorem, for two different proofs see [4] 15].

THEOREM 1 (FUndamentAl THEOREM). Up to an additive logarithmic term

$$
E(x, y)=C(x \mid y)+C(y \mid x) .
$$

Erasing a record $x$ is actually a computation from $x$ to the empty string $\varepsilon$. Hence its irreversibility cost is $E(x, \varepsilon)$.

COROLlaRY 1. Up to a logarithmic additive term, the irreversibility cost of erasure is $E(x, \varepsilon)=C(x)$.

\subsection{Trading Time for Energy}

Because now the time bounds are important we consider the universal Turing machine $U$ to be the machine with two work tapes which can simulate $t$ steps of a multitape Turing machine $T$ in $O(t \log t)$ steps (the Hennie-Stearns simulation). If some multitape Turing machine $T$ computes $x$ in time $t$ from a program $p$, then $U$ computes $x$ in time $O(t \log t)$ from $p$ plus a description of $T$.

Definition 3. Let $C^{t}(x \mid y)$ be the minimal length of binary program (not necessarily reversibly) for the two work tape universal Turing machine $U$ computing $x$ given $y$ (for free) in time t. Formally,

$$
C^{t}(x \mid y)=\min _{p \in \mathcal{N}}\{|p|: U(\langle p, y\rangle)=x \text { in } \leq t(|x|) \text { steps }\} .
$$

$C^{t}(x \mid y)$ is called the $t$-time-limited conditional Kolmogorov complexity of $x$ given $y$. The unconditional version is defined as $C^{t}(x):=$ $C^{t}(x, \varepsilon)$. A program $p$ such that $U(p)=x$ in $\leq t(|x|)$ steps and $|p|=C^{t}(x)$ is denoted as $x_{t}^{*}$.

Note that with $C_{T}^{t}(x \mid y)$ the conditional $t$-time-limited Kolmogorov complexity with respect to Turing machine $T$, for all $x, y, C^{t^{\prime}}(x \mid y) \leq$ $C_{T}^{t}(x \mid y)+c_{T}$, where $t^{\prime}=O(t \log t)$ and $c_{T}$ is a constant depending on $T$ but not on $x$ and $y$.

This $C^{t}(\cdot)$ is the standard definition of time-limited Kolmogorov complexity, [16]. However, in the remainder of the paper we always need to use reversible computations. Fortunately, in $[3]$ it is shown that for any $\varepsilon>0$, ordinary multitape Turing machines using $T$ time and $S$ space can be simulated by reversible ones using time $O(T)$ and space $O\left(S T^{\varepsilon}\right)$.

To do effective erasure of compacted information, we must at the start of the computation provide a time bound $t$. Typically, $t$ is a recursive function and the complexity of its description is small, say $O(1)$. However, in Theorem 2 we allow for very large running times in order to obtain smaller $C^{t}(\cdot)$ values.

THEOREM 2 (EFFECTIVE ERASURE). If $t(|x|) \geq|x|$ is a time bound which is provided at the start of the computation, then erasing an $n$ bit record $x$ by an otherwise reversible computation can be done in time (number of steps) $O\left(2^{|x|} t(|x|)\right)$ at irreversibility cost 
(hence also thermodynamic cost) $C^{t}(x)+2 C^{t}(t \mid x)+4 \log C^{t}(t \mid x)$ bits. (Typically we consider $t$ as some standard explicit time bound and the last two terms adding up to $O(1)$.)

PROOF. Initially we have in memory input $x$ and a program $p$ of length $C^{t}(t, x)$ to compute reversibly $t$ from $x$. To separate binary $x$ and binary $p$ we need to encode a delimiter in at most $2 \log C^{t}(t \mid x)$ bits.

1. Use $x$ and $p$ to reversibly compute $t$. Copy $t$ and reverse the computation. Now we have $x, p$ and $t$.

2. Use $t$ to reversibly dovetail the running of all programs of length less than $x$ to find the shortest one halting in time $t$ with output $x$. This is $x_{t}^{*}$. The computation has produced garbage bits $g\left(x, x_{t}^{*}\right)$. Copy $x_{t}^{*}$, and reverse the computation to obtain $x$ erasing all garbage bits $g\left(x, x_{t}^{*}\right)$. Now we have $x, p, x_{t}^{*}, t$ in memory.

3. Reversibly compute $t$ from $x$ by $p$, cancel one copy of $t$, and reverse the computation. Now we have $x, p, x_{t}^{*}$ in memory.

4. Reversibly cancel $x$ using $x_{t}^{*}$ by the standard method, and then erase $x_{t}^{*}$ and $p$ irreversibly.

Corollary 2. $E(x, \varepsilon) \geq \lim _{t \rightarrow \infty} C^{t}(x)=C(x)$, and by Theorem \up to an additional logarithmic term, $E(x, \varepsilon)=C(x)$.

Essentially, by spending more time we can reduce the thermodynamic cost of erasure of $x_{t}^{*}$ to its absolute minimum. In the limit we spend the optimal value $C(x)$ by erasing $x^{*}$, since $\lim _{t \rightarrow \infty} x_{t}^{*}=x^{*}$. This suggests the existence of a trade-off hierarchy between time and energy. The longer one reversibly computes to perform final irreversible erasures, the less bits are erased and energy is dissipated. This intuitive assertion will be formally stated and rigourously proved below.

DEFINITION 4. Let $U R$ be the reversible version of the two worktape universal Turing machine, simulating the latter in linear time by Bennett's result mentioned above. Let $E^{t}(x, y)$ be the minimum irreversibility cost of an otherwise reversible computation from $x$ to $y$ in time $t$. Formally,

$$
E^{t}(x, y)=\min _{p, q \in \mathcal{N}}\{|p|+|q|: U R(\langle x, p\rangle)=\langle y, q\rangle \text { in } \leq t(|x|) \text { steps }\} \text {. }
$$

Because of the similarity with Corollary $2(E(x, \varepsilon)$ is about $C(x))$ one is erroneously led to believe that $E^{t}(x, \varepsilon)=C^{t}(x)$ up to a log additive term. However, the time-bounds introduce many differences. To reversibly compute $x_{t}^{*}$ we may require (because of the halting problem) at least $O\left(2^{|x|} t(|x|)\right)$ steps after having decoded $t$, as indeed is the case in the proof of Theorem 2 In contrast, $E^{t}(x, \varepsilon)$ is about the number of bits erased in an otherwise reversible computation which uses at most $t$ steps. Therefore, as far as we know possibly $C^{t}(x) \geq E^{t^{\prime}}(x, \varepsilon)$ implies $t^{\prime}=\Omega\left(2^{|x|} t(|x|)\right)$. More concretely, it is easy to see that for each $x$ and $t(|x|) \geq|x|$,

$$
E^{t}(x, \varepsilon) \geq C^{t}(x) \geq E^{t^{\prime}}(x, \varepsilon) / 2,
$$

with $t^{\prime}(|x|)=O\left(t(|x|)\right.$. Theorem 2 can be restated in terms of $E^{t}(\cdot)$ as

$$
E^{t^{\prime}}(x, \varepsilon) \leq C^{t}(x)+2 C^{t}(t \mid x)+4 \log C^{t}(t \mid x),
$$

with $t^{\prime}(|x|)=O\left(2^{|x|} t(|x|)\right)$. Comparing this to the righthand inequality of Equation 1 we have improved the upper bound on erasure cost at the expense of increasing erasure time. However, these bounds only suggest but do not actually prove that we can exchange irreversibility for time. The following result definitely establishes the existence of a trade-off, [15].

Theorem 3 (A Trade-OfF Hierarchy). For each large enough $n$ there is a string $x$ of length $n$ and a sequence of $m=\frac{1}{2} \sqrt{n}$ time functions $t_{1}(n)<t_{2}(n)<\ldots<t_{m}(n)$, such that

$$
E^{t_{1}}(x, \varepsilon)>E^{t_{2}}(x, \varepsilon)>\ldots>E^{t_{m}}(x, \varepsilon) .
$$

In the cost measures like $E^{t}(\cdot, \cdot)$ we have counted both the irreversibly provided and the irreversibly erased bits. But Landauer's principle only charges energy dissipation costs for irreversibly erased bits. It is conceivable that the above results would not hold if one considers as the cost of erasure of a record only the irreversibly erased bits. However, we have show that Theorem 3 also holds for Landauer's dissipation measure, [15], in exactly the same form and by almost the same proof.

\section{OUTLINE SIMULATION RESULTS}

Currently, almost no algorithms and other programs are designed according to reversible principles (and in fact, most tasks like computing Boolean functions are inherently irreversible). To write reversible programs by hand is unnatural and difficult. The natural way is to compile irreversible programs to reversible ones. This raises the question about efficiency of general reversible simulation of irreversible computation. Let us briefly summarize the research reviewed in the next sections.

Suppose the irreversible computation to be simulated uses $T$ time and $S$ space. A first efficient method was proposed by Bennett [3], but it is space hungry and uses ${ }^{2}$ time $S T^{\log 3}$ and space $S \log T$. If $T$ is maximal, that is, exponential in $S$, then the space use is $S^{2}$. This method can be modeled by a reversible pebble game. Reference [17] demonstrated that Bennett's method is optimal for reversible pebble games and that simulation space can be traded off against limited erasing. In [12] it was shown that using a method by Sipser [21] one can reversibly simulate using only $O(S)$ extra space but at the cost of using exponential time. In [9] a relativized separation of reversible and irreversible space-time complexity classes is given.

These previous results seem to suggest that a reversible simulation is stuck with either quadratic space use or exponential time use. This impression turns out to be false [5]:

There is a tradeoff between time and space which has the exponential time simulation and the quadratic space simulation as extremes and for the first time gives a range of simulations using simultaneously subexponential $\left(2^{f(n)}\right.$ is subexponential if $f(n)=$ $o(n))$ time and subquadratic space. The idea is to use Bennett's pebbling game where the pebble steps are intervals of the simulated computation that are bridged by using the exponential simulation method. (It should be noted that embedding Bennett's pebbling game in the exponential method gives no gain, and neither does any other iteration of embeddings of simulation methods.) Careful analysis shows that the simulation using $k$ pebbles takes $T^{\prime}:=S 3^{k} 2^{O\left(T / 2^{k}\right)}$ time and $S^{\prime}=O(k S)$ space, and in some cases the upper bounds are tight. For $k=0$ we have the exponential time simulation method and for $k=\log T$ we have Bennett's method. Interesting values arise for say

(a) $k=\log \log T$ which yields $T^{\prime}=S(\log T)^{\log 3} 2^{O(T / \log T)}$ and $S^{\prime}=S \log \log T \leq S \log S$

\footnotetext{
${ }^{2}$ By judicious choosing of simulation parameters this method can be tweaked to run in $S T^{1+\varepsilon}$ time for every $\varepsilon>0$ at the cost of introducing a multiplicative constant depending on $1 / \varepsilon$. The complexity analysis of [3] was completed in [14].
} 
(b) for $k=\sqrt{\log T}$ we have $S^{\prime}=S \sqrt{\log T} \leq S \sqrt{S}$ and $T^{\prime}=S 3 \sqrt{\log T}$ $2^{O\left(T / 2^{\sqrt{\log T}}\right)}$.

(c) Let $T, S, T^{\prime}, S^{\prime}$ be as above. Eliminating the unknown $k$ shows the tradeoff between simulation time $T^{\prime}$ and extra simulation space $S^{\prime}: T^{\prime}=S 3 \frac{S^{\prime}}{S} 2^{O\left(T / 2^{\frac{S^{\prime}}{S}}\right)}$.

(d) Let $T, S, T^{\prime}, S^{\prime}$ be as above and let the irreversible computation be halting and compute a function from inputs of $n$ bits to outputs. For general reversible simulation by a reversible Turing machine using a binary tape alphabet and a single tape, $S^{\prime} \geq n+$ $\log T+O(1)$ and $T^{\prime} \geq T$. This lower bound is optimal in the sense that it can be achieved by simulations at the cost of using time exponential in $S$.

Main open problem: The ultimate question is whether one can do better, and obtain improved upper and lower bounds on the tradeoff between time and space of reversible simulation, and in particular whether one can have almost linear time and almost linear space simultaneously.

\section{TIME PARSIMONIOUS SIMULATION}

\subsection{Reversible Programming}

Reversible Turing machines or other reversible computers will require special reversible programs. One feature of such programs is that they should be executable when read from bottom to top as well as when read from top to bottom. Examples are the programs we show in the later sections. In general, writing reversible programs will be difficult. However, given a general reversible simulation of irreversible computation, one can simply write an oldfashioned irreversible program in an irreversible programming language, and subsequently simulate it reversibly. This leads to the following:

DEFINITION 5. An irreversible-to-reversible compiler receives an irreversible program as input and reversibly compiles it to a reversible program. Subsequently, the reversible program can be executed reversibly.

Note that there is a decisive difference between reversible circuits and reversible special purpose computers on the one hand, and reversible universal computers on the other hand. While one can design a special-purpose reversible version for every particular irreversible circuit using reversible universal gates, such a method does not yield an irreversible-to-reversible compiler that can execute any irreversible program on a fixed universal reversible computer architecture as we are interested in here.

\subsection{Initial Reversible Simulations}

The reversible simulation in [1] of $T$ steps of an irreversible computation from $x$ to $f(x)$ reversibly computes from input $x$ to output $\langle x, f(x)\rangle$ in $T^{\prime}=O(T)$ time. However, since this reversible simulation at some time instant has to record the entire history of the irreversible computation, its space use increases linearly with the number of simulated steps $T$. That is, if the simulated irreversible computation uses $S$ space, then for some constant $c>1$ the simulation uses $T^{\prime} \approx c+c T$ time and $S^{\prime} \approx c+c(S+T)$ space. This can be an unacceptable amount of space for many practically useful computations.

In [3] another elegant simulation technique is devised reducing the auxiliary storage space. This simulation does not save the entire history of the irreversible computation but it breaks up the simulated computation into segments of about $S$ steps and saves in a hierarchical manner checkpoints consisting of complete instantaneous descriptions of the simulated machine (entire tape con- tents, tape heads positions, state of the finite control). After a later checkpoint is reached and saved, the simulating machine reversibly undoes its intermediate computation, reversibly erasing the intermediate history and reversibly canceling the previously saved checkpoint. Subsequently, the computation is resumed from the new checkpoint onwards. It turns out that this can be done using limited time $T^{\log 3}$ and space $S \log T$. Fine-tuning the method goes as follows: The reversible computation simulates $k^{n}$ segments of length $m$ of irreversible computation in $(2 k-1)^{n}$ segments of length $\Theta(m+S)$ of reversible computation using $n(k-1)+1$ checkpoint registers using $\Theta(m+S)$ space each, for every $k, n, m$.

This way it is established that there are various tradeoffs possible in time-space in between $T^{\prime}=\Theta(T)$ and $S^{\prime}=\Theta(T S)$ at one extreme $(k=1, m=T, n=1)$ and (with the corrections of [14]) $T^{\prime}=$ $\Theta\left(T^{1+\varepsilon} / S^{\varepsilon}\right)$ and $S^{\prime}=\Theta(c(\varepsilon) S(1+\log T / S))$ with $c(\varepsilon)=\varepsilon 2^{1 / \varepsilon}$ for every $\varepsilon>0$, using always the same simulation method but with different parameters $k, n$ where $\varepsilon=\log _{k}(2 k-1)$ and $m=\Theta(S)$. Typically, for $k=2$ we have $\varepsilon=\log 3$. Since for $T>2^{S}$ the machine goes into a computational loop, we always have $S \leq \log T$. Therefore, every irreversible Turing machine using space $S$ can be simulated by a reversible machine using space $S^{2}$ in polynomial time. Let us note that it is possible to improve the situation by reversibly simulating only the irreversible steps. Call a quadruple of a Turing machine irreversible if its range overlaps with the range of another quadruple. A step of the computation is irreversible if it uses an irreversible quadruple. Let the number of irreversible steps in a $T$ step computation be denoted by $I$. Clearly, $I \leq T$. The simulation results hold with $T$ in the auxiliary space use replaced by $I$. In particular, $S^{\prime}=O(S \log I)$. In many computations, $I$ may be much smaller than $T$. There arises the problem of estimating the number of irreversible steps in a computation. (More complicatedly, one could extend the notion of irreversible step to those steps which can be reversed on local information alone. In some cases this is possible even when the used quadruple itself was irreversible.)

We at some point conjectured that all reversible simulations of an irreversible computation can essentially be represented as the pebble game defined below, and that consequently the lower bound of Corollary 4 applies to all reversible simulations of irreversible computations. This conjecture was refuted in [12] using a technique due to [21] to show that there exists a general reversible simulation of an irreversible computation using only order $S$ space at the cost of using a thoroughly unrealistic simulation time exponential in $S$.

In retrospect the conjecture was phrased too general: it should be restricted to $u$ seful simulations - using linear or slightly superlinear time and space simultaneously. The real question is whether there is a compiler that takes as input any irreversible algorithm $A$ using $S$ space and $T$ time and produces a reversible algorithm $B$ such that $B(x)=A(x)$ for all input $x$ and using $T^{\prime}=O(T)$ time and $S^{\prime}=O(S)$ space. In the extreme cases of time and space use this is possible: If $S=\Theta(T)$ then the simulation in $\llbracket 1]$ does the trick, and if $T=\Theta\left(2^{S}\right)$ then the simulation of [12] works. For all other cases the pebble game analysis below has been used in [9] to show that any such simulation, if it exists, cannot relativize to oracles, or work in cases where the space bound is much less than the input length. (This is a standard method of giving evidence that the aimed-for resulthere: simulation doesn't exist-is likely to be true in case the result itself is too hard to obtain.)

\subsection{Reversible Pebbling}

Let $G$ be a linear list of nodes $\left\{1,2, \ldots, T_{G}\right\}$. We define a pebble game on $G$ as follows. The game proceeds in a discrete sequence of steps of a single player. There are $n$ pebbles which can be put on nodes of $G$. At any time the set of pebbles is divided in pebbles on 
nodes of $G$ and the remaining pebbles which are called free pebbles. At every step either an existing free pebble can be put on a node of $G$ (and is thus removed from the free pebble pool) or be removed from a node of $G$ (and is added to the free pebble pool). Initially $G$ is unpebbled and there is a pool of free pebbles. The game is played according to the following rule:

Reversible Pebble Rule: If node $i$ is occupied by a pebble, then one may either place a free pebble on node $i+1$ (if it was not occupied before), or remove the pebble from node $i+1$.

We assume an extra initial node 0 permanently occupied by an extra, fixed pebble, so that node 1 may be (un)pebbled at will. This pebble game is inspired by the method of simulating irreversible Turing Machines on reversible ones in a space efficient manner. The placement of a pebble corresponds to checkpointing the next state of the irreversible computation, while the removal of a pebble corresponds to reversibly erasing a checkpoint. Our main interest is in determining the number of pebbles $k$ needed to pebble a given node $i$.

The maximum number $n$ of pebbles which are simultaneously on $G$ at any one time in the game gives the space complexity $n S$ of the simulation. If one deletes a pebble not following the above rules, then this means a block of bits of size $S$ is erased irreversibly. This pebble game is inspired by the method of simulating irreversible Turing Machines on reversible ones in a space efficient manner. The placement of a pebble corresponds to checkpointing the current state of the irreversible computation, while the removal of a pebble corresponds to reversibly erasing a checkpoint. Our main interest is in determining the number of pebbles $k$ needed to pebble a given node $i$.

The maximum number $n$ of pebbles which are simultaneously on $G$ at any one time in the game gives the space complexity $n S$ of the simulation. If one deletes a pebble not following the above rules, then this means a block of bits of size $S$ is erased irreversibly. The limitation to Bennett's simulation is in fact space, rather than time. When space is limited, we may not have enough place to store garbage, and these garbage bits will have to be irreversibly erased. We establish a tight lower bound for any strategy for the pebble game in order to obtain a space-irreversibility tradeoff.

\subsection{Algorithm}

We describe the idea of Bennett's simulation [3]. This simulation is optimal [17] among all reversible pebble games, and we will show the proof below. The total computation of $T$ steps is broken into $2^{k}$ segments of length $m=T 2^{-k}$. Every $m$ th point of the computation is a node in the pebbling game; node $i$ corresponding to im steps of computation.

For each pebble a section of tape is reserved long enough to store the whole configuration of the simulated machine. By enlarging the tape alphabet, each pebble will require space only $S+O(1)$.

Both the pebbling and unpebbling of a pebble $t$ on some node, given that the previous node has a pebble $s$ on it, will be achieved by a single reversible procedure bridge $(s, t)$. This looks up the configuration at section $s$, simulates $m$ steps of computation in a manner described in section 6 and exclusive-or's the result into section $t$. If $t$ was a free pebble, meaning that its tape section is all zeroes, the result is that pebble $t$ occupies the next node. If $t$ already pebbled that node then it will be zeroed as a result.

The essence of Bennett's simulation is a recursive subdivision of a computation path into 2 halves, which are traversed in 3 stages; the first stage gets the midpoint pebbled, the second gets the endpoint pebbled, and the 3 rd recovers the midpoint pebble. The following recursive procedure implements this scheme; $\operatorname{Pebble}(s, t, n)$ uses free pebbles $0, \ldots, n-1$ to compute the $2^{n}$ th node after the one pebbled by $s$, and exclusive-or's that node with pebble $t$ (either putting $t$ on the node or taking it off). Its correctness follows by straightforward induction. Note that it is its own reverse; executing it twice will produce no net change. The pebble parameters $s$ and $t$ are simply numbers in the range $-1,0,1, \ldots, k$. Pebble -1 is permanently on node 0 , pebble $k$ gets to pebble the final node, and pebble $i$, for $0 \leq i<k$ pebbles nodes that are odd multiples of $2^{i}$. The entire simulation is carried out with a call pebble $(-1, k, k)$.

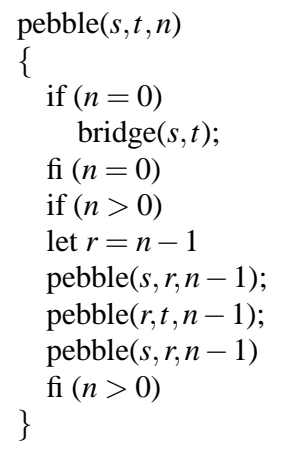

As noted by Bennett, both branches and merges must be labeled with mutually exclusive conditions to ensure reversibility. Recursion can be easily implemented reversibly by introducing an extra stack tape, which will hold at most $n$ stack frames of size $O(\log n)$ each, for a total of $O(n \log n)$.

\subsection{Optimality}

This pebbling method is optimal in that no more than $2^{n+1}-1$ steps can be bridged with $n$ pebbles [17]. It is easy to see that the method achieves this; the difficult part is showing that no reversible pebbling method can do better. It turns out that characterizing the maximum node that can be pebbled with a given number of pebbles is best done by completely characterizing what pebble configurations are realizable. First we need to introduce some helpful notions.

In a given pebble configuration with $f$ free pebbles, a placed pebble is called available if there is another pebble at most $2^{f}$ positions to its left ( 0 being the leftmost node). According to the above procedures, an available pebble can be removed with the use of the free pebbles. For convenience we imagine this as a single big step in our game.

Call a pebble configuration weakly solvable if there is a way of repeatedly removing an available pebble until all are free. Note that such configurations are necessarily realizable, since the removal process can be run in reverse to recreate the original configuration. Call a pebble configuration strongly solvable if all ways of repeatedly removing an available pebble lead to all being free. Obviously any strongly solvable configuration is also weakly solvable.

The starting configuration is obviously both weakly and strongly solvable. How does the single rule of the game affect solvability? Clearly, adding a pebble to a weakly solvable configuation yields another weakly solvable configuation, while removing a pebble from a strongly solvable configuation yields another strongly solvable configuation. It is not clear if removing a pebble from a weakly solvable configuation yields another one. If such is the case then we may conclude that all realizable configurations are weakly solvable and hence the two classes coincide. This is exactly what the next theorem shows.

THEOREM 4. Every weakly solvable configuration is strongly solvable. 
PROOF. Let $f$ be the number of free pebbles in a weakly solvable configuration. Number the placed pebbles $f, f+1, \ldots, n-1$ according to their order of removal. It is given that, for all $i$, pebble $i$ has a higher-numbered pebble at most $2^{i}$ positions to its left (number the fixed pebble at 0 infinity). We know that pebble $f$ is available. Suppose a pebble $g$ with $g>f$ is also available-so there must be a pebble at most $2^{f}$ positions to its left. It suffices to show that if pebble $g$ is removed first, then pebbles $f, f+1, \ldots, g-1$ are still available when their turn comes. Suppose pebble $j$ finds pebble $g$ at most $2^{j}$ places to its left (otherwise $j$ will still be available after $g$ 's removal for sure). Then after removal of pebbles $g, f, f+1, \ldots, j-1$, it will still find a higher-numbered pebble at $\operatorname{most} 2^{j}+2^{f}+2^{f}+2^{f+1}+\cdots+2^{j-1} \leq 2^{j+1}$ places to its left, thus making it available given the extra now free pebble $g$.

COROLlary 3. A configuration with $f$ free pebbles is realizable if and only if its placed pebbles can be numbered $f, f+$ $1, \ldots, n-1$ such that pebble $i$ has a higher-numbered pebble at most $2^{i}$ positions to its left.

COROLlaRY 4. The maximum reachable node with $n$ pebbles is $\sum_{i=0}^{n-1} 2^{i}=2^{n}-1$.

Moreover, if pebble $(s, n)$ takes $t(n)$ steps we find $t(0)=1$ and $t(n)=3 t(n-1)+1=\left(3^{n+1}-1\right) / 2$. That is, the number of steps $T_{G}^{\prime}$ of a winning play of a pebble game of size $T_{G}=2^{n}-1$ is $T_{G}^{\prime} \approx$ $1.53^{n}$, that is, $T_{G}^{\prime} \approx T_{G}^{\log 3}$.

\subsection{Trading Space for Erasures}

The simulation above follows the rules of the pebble game of length $T_{G}=2^{n}-1$ with $n$ pebbles above. A winning strategy for a game of length $T_{G}$ using $n$ pebbles corresponds with reversibly simulating $T_{G}$ segments of $S$ steps of an irreversible computation using $S$ space such that the reversible simulator uses $T^{\prime} \approx S T_{G}^{\prime} \approx S T_{G}^{\log 3}$ steps and total space $S^{\prime}=n S$. The space $S^{\prime}$ corresponds to the maximal number of pebbles on $G$ at any time during the game. The placement or removal of a pebble in the game corresponds to the reversible copying or reversible cancelation of a 'checkpoint' consisting of the entire instantaneous description of size $S$ (work tape contents, location of heads, state of finite control) of the simulated irreversible machine. The total time $T_{G} S$ used by the irreversible computation is broken up in segments of size $S$ so that the reversible copying and canceling of a checkpoints takes about the same number of steps as the computation segments in between checkpoints. 3

We can now formulate a tradeoff between space used by a polynomial time reversible computation and irreversible erasures as proposed in [17]. First we show that allowing a limited amount of erasure in an otherwise reversible computation means that we can get by with less work space. Therefore, we define an $m$-erasure pebble game as the pebble game above but with the additional rule

- In at most $m$ steps the player can remove a pebble from any node $i>1$ without node $i-1$ being pebbled at the time.

An $m$-erasure pebble game corresponds with an otherwise reversible computation using $m S$ irreversible bit erasures, where $S$ is the space used by the irreversible computation being simulated.

\footnotetext{
${ }^{3}$ If we are to account for the permanent pebble on node 0 , we get that the simulation uses $n+1$ pebbles for a pebble game with $n$ pebbles of length $T_{G}+1$. The simulation uses $n+1=S^{\prime} / S$ pebbles for a simulated number of $S\left(T_{G}+1\right)$ steps of the irreversible computation.
}

LEMMA 2. There is a winning strategy with $n+2$ pebbles and $m-1$ erasures for pebble games $G$ with $T_{G}=m 2^{n}$, for all $m \geq 1$.

PROOF. The strategy is to use 2 pebbles as springboards that are alternately placed $2^{n}$ in front of each other using the remaining $n$ pebbles to bridge the distance. The most backward springboard can be erased from its old position once all $n$ pebbles are cleared from the space between it and the front springboard.

The simulation time $T_{G}^{\prime}$ is $T_{G}^{\prime} \approx 2 m \cdot 3^{n-1}+2 \approx 2 m\left(T_{G} / m\right)^{\log 3}=$ $2 m^{1-\log 3} T_{G}^{\log 3}$ for $T_{G}=m 2^{n-1}$.

Theorem 5 (SPACE-IRreVERsibility). (i) Pebble games $G$ of size $2^{n}-1$ can be won using $n$ pebbles but not using $n-1$ pebbles.

(ii) If $G$ is a pebble game with a winning strategy using $n$ pebbles without erasures, then there is also a winning strategy for $G$ using $E$ erasures and $n-\log (E+1)$ pebbles (for $E$ is an odd integer at least 1).

Proof. (i) By Corollory 4

(ii) By (i), $T_{G}=2^{n}-1$ is the maximum length of a pebble game $G$ for which there is a winning strategy using $n$ pebbles and no erasures. By Lemma 2 we can pebble a game $G$ of length $T_{G}=$ $m 2^{n-\log m}=2^{n}$ using $n+1-\log m$ pebbles and $2 m-1$ erasures.

We analyze the consequences of Theorem 5 It is convenient to consider the special sequence of values $E:=2^{k+2}-1$ for $k:=$ $0,1, \ldots$ Let $G$ be Bennett's pebble game of Lemma 4 of length $T_{G}=2^{n}-1$. It can be won using $n$ pebbles without erasures, or using $n-k$ pebbles plus $2^{k+2}-1$ erasures (which gives a gain over not erasing as in Lemma 4 only for $k \geq 1$ ), but not using $n-1$ pebbles.

Therefore, we can exchange space use for irreversible erasures. Such a tradeoff can be used to reduce the space requirements of the reversible simulation. The correspondence between the erasure pebble game and the otherwise reversible computations using irreversible erasures is that if the pebble game uses $n-k$ pebbles and $2^{k+2}-1$ erasures, then the otherwise reversible computation uses $(n-k) S$ space and erases $\left(2^{k+2}-1\right) S$ bits irreversibly.

Therefore, a reversible simulation according to the pebble game of every irreversible computation of length $T=\left(2^{n}-1\right) S$ can be done using $n S$ space using $(T / S)^{\log 3} S$ time, but is impossible using $(n-1) S$ space. It can also be performed using $(n-k) S$ space, $\left(2^{k+2}-1\right) S$ irreversible bit erasures and $2^{(k+1)(1-\log 3)+1}(T / S)^{\log 3} S$ time. In the extreme case we use no space to store the history and erase about $4 T$ bits. This corresponds to the fact that an irreversible computation may overwrite its scanned symbol irreversibly at each step.

DEFINITION 6. Consider a simulation according to the pebble game using $S^{\prime}$ storage space and $T^{\prime}$ time which reversibly computes $y=\langle x, f(x)\rangle$ from $x$ in order to simulate an irreversible computation using $S$ storage space and T time which computes $f(x)$ from $x$. The irreversible simulation cost $B^{S^{\prime}}(x, y)$ of the simulation is the number of irreversibly erased bits in the simulation (with the parameters $S, T, T^{\prime}$ understood).

If the irreversible simulated computation from $x$ to $f(x)$ uses $T$ steps, then for $S^{\prime}=n S$ and $n=\log (T / S)$ we have above treated the most space parsimonious simulation which yields $B^{S^{\prime}}(x, y)=0$, with $y=\langle x, f(x)\rangle$.

COROLlary 5. Simulating a $T=\left(2^{n}-1\right) S$ step irreversible computation from $x$ to $f(x)$ using $S$ space by a computation from $x$ to $y=\langle x, f(x)\rangle$, the irreversible simulation cost satisfies: 
(i) $B^{(n-k) S}(x, y) \leq B^{n S}(x, y)+\left(2^{k+2}-1\right) S$, for $n \geq k \geq 1$.

(ii) $B^{(n-1) S}(x, y)>B^{n S}(x, y)$, for $n \geq 1$.

For the most space parsimonious simulation with $n=\log (T / S)$ this means that

$$
B^{S(\log (T / S)-k)}(x, y) \leq B^{S \log (T / S)}(x, y)+\left(2^{k+2}-1\right) S .
$$

\section{SPACE PARSIMONIOUS SIMULATION}

Lange, McKenzie and Tapp, [12], devised a reversible simulation, LMT-simulation for short, that doesn't use extra space, at the cost of using exponential time. Their main idea of reversibly simulating a machine without using more space is by reversibly cycling through the configuration tree of the machine (more precisely the connected component containing the input configuration). This configuration tree is a tree whose nodes are the machine configurations and where two nodes are connected by an edge if the machine moves in one step from one configuration to the other. We consider each edge to consist of two half-edges, each adjacent to one configuration.

The configuration tree can be traversed by alternating two permutations on half-edges: a swapping permutation which swaps the two half-edges constituting each edge, and a rotation permutation whose orbits are all the half-edges adjacent to one configuration. Both permutations can be implemented in a constant number of steps. For simplicity one assumes the simulated machine strictly alternates moving and writing transitions. To prevent the simulation from exceeding the available space $S$, each pebble section is marked with special left and right markers $\dagger, \ddagger$, which we assume the simulated machine not to cross. Since this only prevents crossings in the forward simulation, we furthermore, with the head on the left (right) marker, only consider previous moving transitions from the right (left).

\section{TIME-SPACE TRADEOFF}

A call pebble $(s, t, n)$ results in $3^{n}$ calls to bridge $(\cdot, \cdot)$. Bennett chose the number of pebbles large enough $(n=\Omega(\log T))$ so that $m$ becomes small, on the order of the space $S$ used by the simulated machine. In that case bridge $(s, t)$ is easily implemented with the help of an additional history tape of size $m$ which records the sequence of transitions. Instead, [5] showed how to allow an arbitrary choice of $n$ and resort to the space efficient simulation of [12] to bridge the pebbled checkpoints. A similar solution was arrived at later and independently in the unpublished manuscript [22].

To adapt the LMT simulation to our needs, we equip our simulating machine with one extra tape to hold the simulated configuration and another extra tape counting the difference between forward and backward steps simulated. $m=2^{n}$ steps of computation can be bridged with a $\log m$ bits binary counter, incremented with each simulated forward step, and decremented with each simulated backward step. Having obtained the configuration $m$ steps beyond that of pebble $s$, it is exclusive-or'd into section $t$ and then the LMT simulation is reversed to end up with a zero counter and a copy of section $s$, which is blanked by an exclusive-or from the original.

\section{bridge $(s, t)$}

\{

copy section $s$ onto (blanked) simulation tape

setup: goto enter;

loop1: come from endloop1;

simulate step with swap\&rotate and adjust counter

if (counter $=0$ )

rotate back;

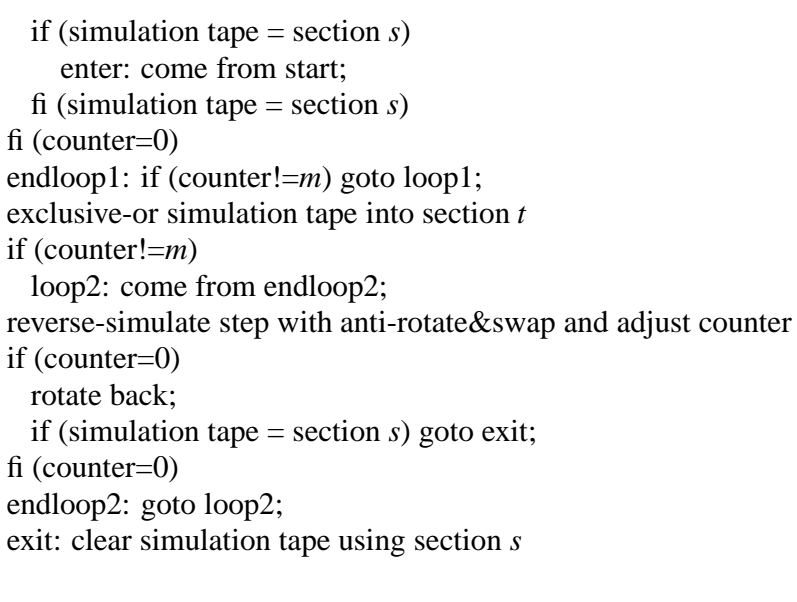

\subsection{Complexity Analysis}

Let us analyze the time and space used by this simulation.

THEOREM 6. An irreversible computation using time $T$ and space $S$ can be simulated reversibly in time $T^{\prime}=3^{k} 2^{O\left(T / 2^{k}\right)} S$ and space $S^{\prime}=S(1+O(k))$, where $k$ is a parameter that can be chosen freely $0 \leq k \leq \log T$ to obtain the required tradeoff between reversible time $T^{\prime}$ and space $S^{\prime}$.

PROOF. (Sketch) Every invocation of the bridge() procedure takes time $O\left(2^{\dot{O}(\mathrm{~m})} \mathrm{S}\right)$. That is, every configuration has at most $O(1)$ predecessor configurations where it can have come from (constant number of states, constant alphabet size and choice of direction). Hence there are $\leq 2^{O(m)}$ configurations to be searched and about as many potential start configurations leading in $m$ moves to the goal configuration, and every tape section comparison takes time $O(S)$. The pebbling game over $2^{k}$ nodes takes $3^{k}$ (un)pebbling steps each of which is an invocation of bridge(). Filling in $m=T / 2^{k}$ gives the claimed time bound. Each of the $k+O(1)$ pebbles takes space $O(S)$, as does the simulation tape and the counter, giving the claimed total space.

It is easy to verify that for some simulations the upper bound is tight. The boundary cases, $k=0$ gives the LMT-simulation using exponential time and no extra space, and $k=\log T$ gives Bennett's simulation using at most square space and subquadratic time. Taking intermediate values of $k$ we can choose to reduce time at the cost of an increase of space use and vice versa. In particular, special values $k=\log \log T$ and $k=\sqrt{T}$ give the results using simultaneously subexponential time and subquadratic space exhibited in the introduction. Eliminating $k$ we obtain:

COROLlary 6. Let $T, S, T^{\prime}, S^{\prime}$ be as above. Then there is a reversible simulation that has the following tradeoff between simulation time $T^{\prime}$ and extra simulation space $S^{\prime}$ :

$$
T^{\prime}=S 3 \frac{S^{\prime}}{S} 2^{O\left(T / 2^{\frac{S^{\prime}}{S}}\right)}
$$

\subsection{Local Irreversible Actions}

Suppose we have an otherwise reversible computation containing local irreversible actions. Then we need to reversibly simulate only the subsequence of irreversible steps, leaving the connecting reversible computation segments unchanged. That is, an irreversiblity parsimonious computation is much cheaper to reversibly simulate than an irreversibility hungry one. 


\section{UNKNOWN COMPUTING TIME}

In the previous analysis we have tacitly assumed that the reversible simulator knows in advance the number of steps $T$ taken by the irreversible computation to be simulated. In this context one can distinguish on-line computations and off-line computations to be simulated. On-line computations are computations which interact with the outside environment and in principle keep running forever. An example is the operating system of a computer. Off-line computations are computations which compute a definite function from an input (argument) to an output (value). For example, given as input a positive integer number, compute as output all its prime factors. For every input such an algorithm will have a definite running time.

There is a well-known simple device to remove this dependency for batch computations without increasing the simulation time (and space) too much [17]. Suppose we want to simulate a computation with unknown computation time $T$. Then we simulate $t$ steps of the computation with $t$ running through the sequence of values $2,2^{2}, 2^{3}, \ldots$ For every value $t$ takes on we reversibly simulate the first $t$ steps of the irreversible computation. If $T>t$ then the computation is not finished at the end of this simulation. Subsequently we reversibly undo the computation until the initial state is reached again, set $t:=2 t$ and reversibly simulate again. This way we continue until $t \geq T$ at which bound the computation finishes. The total time spent in this simulation is

$$
T^{\prime \prime} \leq 2 \sum_{i=1}^{\lceil\log T\rceil} S 3^{\frac{S^{\prime}}{S}} 2^{O\left(2^{i-\frac{S^{\prime}}{S}}\right)} \leq 2 T^{\prime} .
$$

\section{LOWER BOUND}

It is not difficult to show a simple lower bound [5] on the extra storage space required for general reversible simulation. We consider only irreversible computations that are halting computations performing a mapping from an input to an output. For convenience we assume that the Turing machine has a single binary work tape delemited by markers $\dagger, \ddagger$ that are placed $S$ positions apart. Initially the binary input of length $n$ is written left adjusted on the work tape. At the end of the computation the output is written left adjusted on the work tape. The markers are never moved. Such a machine clearly can perform every computation as long as $S$ is large enough with respect to $n$. Assume that the reversible simulator is a similar model albeit reversible. The average number of steps in the computation is the uniform average over all equally likely inputs of $n$ bits.

THEOREM 7. To generally simulate an irreversible halting computation of a Turing machine as above using storage space $S$ and $T$ steps on average, on inputs of length $n$, by a general reversible computation using $S^{\prime}$ storage space and $T^{\prime}$ steps on average, the reversible simulator Turing machine having $q^{\prime}$ states, requires trivially $T^{\prime} \geq T$ and $S^{\prime} \geq n+\log T-O(1)$ up to a logarithmic additive term.

Proof. There are $2^{n}$ possible inputs to the irreversible computation, the computation on every input using on average $T$ steps. A general simulation of this machine cannot use the semantics of the function being simulated but must simulate every step of the simulated machine. Hence $T^{\prime} \geq T$. The simulator being reversible requires different configurations for every step of everyone of the simulated computations that is, at least $2^{n} T$ configurations. The simulating machine has not more than $q^{\prime} 2^{S^{\prime}} S^{\prime}$ distinct configurations $-2^{S^{\prime}}$ distinct values on the work tape, $q^{\prime}$ states, and $S^{\prime}$ head positions for the combination of input tape and work tape.
Therefore, $q^{\prime} 2^{S^{\prime}} S^{\prime} \geq 2^{n} T$. That is, $q^{\prime} S^{\prime} 2^{S^{\prime}-n} \geq T$ which shows that $S^{\prime}-n-\log S^{\prime} \geq \log T-\log q^{\prime}$.

For example, consider irreversible computations that don't use extra space apart from the space to hold the input, that is, $S=n$. An example is the computation of $f(x)=0$.

- If $T$ is polynomial in $n$ then $S^{\prime}=n+\Omega(\log n)$.

- If $T$ is exponential in $n$ then $S^{\prime}=n+\Omega(n)$.

Thus, in some cases the LMT-algorithm is required to use extra space if we deal with halting computations computing a function from input to output. In the final version of the paper [12] the authors have added that their simulation uses some extra space for counting (essentially $O(S)$ ) in case we require halting computations from input to output, matching the lower bound above for $S=n$ since their simulation uses on average $T^{\prime}$ steps exponential in $S$.

Optimality and Tradeoffs: The lower bound of Theorem $\square$ is optimal in the following sense. As one extreme, the LMT-algorithm of [12] discussed above uses $S^{\prime}=n+\log T$ space for simulating irreversible computations of total functions on inputs of $n$ bits, but at the cost of using $T^{\prime}=\Omega\left(2^{S}\right)$ simulation time. As the other extreme, Bennett's simple algorithm in [1] uses $T^{\prime}=O(T)$ reversible simulation time, but at the cost of using $S^{\prime}=\Omega(T)$ additional storage space. This implies that improvements in determining the complexity of reversible simulation must consider time-space tradeoffs.

\section{REFERENCES}

[1] C.H. Bennett. Logical reversibility of computation. IBM J. Res. Develop., 17:525-532, 1973.

[2] C.H. Bennett. The thermodynamics of computation-a review. Int. J. Theoret. Phys., 21(1982), 905-940.

[3] C.H. Bennett. Time-space tradeoffs for reversible computation. SIAM J. Comput., 18(1989), 766-776.

[4] C.H. Bennett, P. Gács, M. Li, P.M.B. Vitányi, and W. Zurek. Information Distance, IEEE Transactions on Information Theory, 44:4(1998), 1407-1423.

[5] H. Buhrman, J. Tromp, P. Vitanyi, Time and space bounds for reversible simulation, Journal of Physics A: Mathematical and General, 34:35(2001), 6821-6830.

[6] R.P. Feynman, Simulating physics with computers, Int. J. Theoret. Physics, 21(1982), 467-488;

[7] E. Fredkin and T. Toffoli. Conservative logic. Int. J. Theoret. Phys., 21(1982),219-253.

[8] M. Frank, T. Knight, and N. Margolus, Reversibility in optimally scalable computer architectures, Manuscript, MIT-LCS, 1997 // http://www.ai.mit.edu/ mpf/publications.html

[9] M.P. Frank and M.J. Ammer, Separations of reversible and irreversible space-time complexity classes, Submitted.// http://www.ai.mit.edu/ mpf/rc/memos/M06_oracle.html

[10] R.W. Keyes, IBM J. Res. Dev., 32(1988), 24-28.

[11] R. Landauer. Irreversibility and heat generation in the computing process. IBM J. Res. Develop., 5:183-191, 1961.

[12] K.J. Lange, P. McKenzie, and A. Tapp, Reversible space equals deterministic space, J. Comput. System Sci., 60:2(2000), 354-367.

[13] Y. Lecerf, Machines de Turing réversibles. Récursive insolubilité en $n \in N$ de l'équation $u=\theta^{n}$, où $\theta$ est un "isomorphisme de codes", Comptes Rendus, 257(1963), 2597-2600. 
[14] R.Y. Levine and A.T. Sherman, A note on Bennett's time-space tradeoff for reversible computation, SIAM J. Comput., 19:4(1990), 673-677.

[15] M. Li and P.M.B. Vitányi, Reversibility and adiabatic computation: trading time and space for energy, Proc. Royal Society of London, Series A, 452(1996), 769-789.

[16] M. Li and P.M.B. Vitányi. An Introduction to Kolmogorov Complexity and its Applications, Springer-Verlag, New York, 2nd Edition, 1997.

[17] M. Li, J. Tromp, and P. Vitányi, Reversible simulation of irreversible computation. Physica D, 120(1998) 168-176.
[18] K. Morita, A. Shirasaki, and Y. Gono, A 1-tape 2-symbol reversible Turing machine, IEEE Trans. IEICE, E72 (1989), 223-228.

[19] M. Nielsen, I. Chuang, Quantum Computation and Quantum Information, Cambridge University Press, 2000.

[20] P.W. Shor, Polynomial-time algorithms for prime factorization and discrete logarithms on a quantum computer. SIAM J. Comput., 26:5(1997), 1484-1509.

[21] M. Sipser, Halting space-bounded computation, Theoret. Comp. Sci., 10(1990), 335-338.

[22] Ryan Williams, Space-Efficient Reversible Simulations, DIMACS REU report, July 2000. URL

http://dimacs.rutgers.edu/ ryanw/ 